\title{
Polyethyleneglycol an efficient solvent for stereoselective synthesis of $\beta$-amino ketones via direct Mannich reaction
}

\author{
Mohammad A. Bigdeli, ${ }^{\text {a }}$ Majid M. Heravi, ${ }^{\text {b }}$ Firouzeh Nemati, ${ }^{\text {** }}$ \\ and Gholam Hossein Mahdavinia ${ }^{c}$ \\ ${ }^{a}$ Faculty of Chemistry, Teacher Training University, 49 Mofateh St., Tehran, Iran \\ ${ }^{b}$ Departemant of Chemistry, School of Science, Azzahra University, Vanak, Tehran, Iran \\ ${ }^{c}$ Department of Chemistry, Islamic Azad University-Marvdasht Branch, Marvdasht, Iran \\ E-mail:firouzehnemati@yahoo.com
}

\begin{abstract}
Polyethyleneglycol- 2,4,6-Trichloro-1,3,5-Triazine (PEG 400-TCT) promotes a series of three component one-pot reactions of cyclohexanones with aromatic aldehydes and aniline. $\beta$-amino ketones are afforded in high yields and high stereoselectivity, in favor of the anti-isomer. Four new compounds are reported.
\end{abstract}

Keywords: Polyethyleneglycol, 2,4,6-trichloro-1,3,5-triazine, stereoselective, three component Mannich reaction

\section{Introduction}

The search for new reaction media to replace volatile and often toxic solvents that are used in organic synthetic procedures is an important objective of significant environmental consequence. While the use of water as solvent is probably the most desirable approach, this is often not possible due to the hydrophobic nature of the reactants and the sensitivity of many catalysts to aqueous conditions. In this manuscript we describe the use of a widely available polymer, polyethyleneglycol (PEG 400), as non-toxic, inexpensive and non-volatile solvent for the stereoselective synthesis of $\beta$-amino ketones via one-pot Mannich reactions.

Mannich reactions are among the most important carbon-carbon bond forming reactions in organic synthesis. ${ }^{1}$ They provide $\beta$-amino carbonyl compounds, which are important synthetic intermediates for various pharmaceuticals and natural products. ${ }^{2}$ A more desirable route for this reaction is the use of a one-pot three-component strategy that allows for a wide range of structural variations.

In this context, recent developments of asymmetric synthesis, using a three-component protocol, have made the Mannich reaction even more valuable. ${ }^{3}$ Recently, direct Mannich 
reaction of aldehydes, ketones and arylamines via Lewis acids ${ }^{4}$, lanthanides ${ }^{5}$, transition metal salt catalyst ${ }^{6}$, and organic catalytic ${ }^{7}$ are reported. Most of these methods, however, suffer from severe drawbacks including the use of organic solvents, expensive reagents or catalysts, long reaction times, low yields and use of special techniques for catalyst preparation. Hence, there is still considerable high interest in developing convenient methods for the synthesis of $\beta$-amino ketones.

\section{Results and Discussion}

In pursuit of environmentally friendly methods of preparation of chemical compounds, we decided to look at the use of polyethyleneglycol as an inexpensive, non-volatile and commercially available solvent for the synthesis of $\beta$-amino ketones at room temperature. We use from 2,4,6-Trichloro-1,3,5-Triazine (cyanuric chloride, TCT)(Scheme 1), as a cheap and commercially available reagent. ${ }^{8}$ It performed as a dry acid catalyst source that required in Mannich reactions.

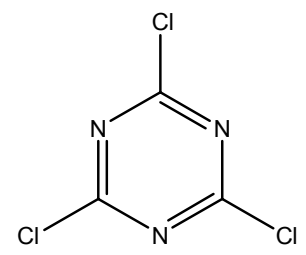

Scheme 1. TCT structure.

TCT generates $\mathrm{HCl}$ (insitu) and polyethyleneglycol acts as an appropriate solvent, a green solvent in which dry $\mathrm{HCl}$ is active and all products precipitate out readily. The combination of the two, gave remarkable results. TCT alone in $\mathrm{H}_{2} \mathrm{O}$ or ethanol gave no reaction. Polyethyleneglycol alone did not produce any results either (Scheme 2).<smiles>[R]CC(C)C(C)C1CCC(=O)CC1</smiles>

Scheme 2. The one-pot Mannich reaction. 
TCT $(1.37 \%$ relative to arylaldehyde $)(0.005 \mathrm{~g}, 0.027 \mathrm{mmol})$ was found to be the optimum amount needed for this transformation resulting in higher conversions and lower reaction times. (Table1).

Table 1. TCT catalyzed the Mannich type reactions of cyclohexanone, benzaldehydes and aniline in PEG 400

\begin{tabular}{lll}
\hline Entry & TCT g (mmol) & Yields (\%) \\
\hline 1 & $0.001(0.005)$ & 85 \\
2 & $0.003(0.016)$ & 85 \\
3 & $0.005(0.027)$ & 99 \\
4 & $0.007(0.037)$ & 80 \\
5 & $0.01(0.054)$ & 50 \\
\hline
\end{tabular}

In all cases under study, it was found that reactions proceeded with almost complete antiselectivity and high yields (Table 2). We found that substituted anilines and benzaldehydes with electron-withdrawing groups and strong electron-releasing groups did not work well. The results are summarized in Table 2.

Table 2. Direct Mannich-type reaction of aromatic aldehydes, aniline and cyclohexanone or 4methylcyclohexanone ${ }^{\text {a }}$

\begin{tabular}{llllll}
\hline Entry & $\mathrm{R}$ & $\mathrm{Ar}$ & Time (h) & Yield (\%) $^{\mathrm{b}}$ & anti/syn $^{\mathrm{c}}$ \\
\hline 1 & $\mathrm{H}$ & $\mathrm{Ph}$ & 1 & 99 & $99: 1$ \\
2 & $\mathrm{H}$ & 4- $\mathrm{MeC}_{6} \mathrm{H}_{4}$ & 2 & 85 & $99: 1$ \\
3 & $\mathrm{H}$ & 1-naphthyl & 1.5 & 99 & $99: 1$ \\
4 & $\mathrm{H}$ & 2-naphthyl & 1.5 & 99 & $99: 1$ \\
5 & $\mathrm{H}$ & 2- $\mathrm{ClC}_{6} \mathrm{H}_{4}$ & 2.5 & 75 & $99: 1$ \\
6 & $\mathrm{Me}$ & $\mathrm{Ph}$ & 1 & 99 & $95: 5$ \\
7 & $\mathrm{Me}$ & 1-naphthyl & 2 & 98 & $99: 1$ \\
8 & $\mathrm{Me}$ & 2-naphthyl & 1 & 99 & $99: 1$ \\
9 & $\mathrm{Me}$ & 2-ClC $_{6} \mathrm{H}_{4}$ & 2 & 70 & $99: 1$ \\
\hline
\end{tabular}

${ }^{a}$ Reaction condition: aromatic aldehyde $(2 \mathrm{mmol})$, aniline $(2 \mathrm{mmol})$, cyclohexanone or 4methylcyclohexanone $(2.2 \mathrm{mmol})$, catalyst $(1.37 \mathrm{~mol} \%)$.

${ }^{\mathrm{b}}$ Isolated yields, products were confirmed by IR, ${ }^{1} \mathrm{H}$ NMR and ${ }^{13} \mathrm{C}$ NMR.

${ }^{\mathrm{c}}$ Anti/syn ratio was determined by ${ }^{1} \mathrm{H}$ NMR.

The anti- and syn- isomers were identified by the coupling constants $(J)$ of the vicinal protons adjacent to $\mathrm{C}=\mathrm{O}$ and $\mathrm{NH}$ in ${ }^{1} \mathrm{H}$ NMR spectra. ${ }^{9}$ The coupling constants for anti- isomers are reported to be bigger than those of $s y n$-isomers. ${ }^{10}$ 
We can conclude that interaction between solvent or catalyst with the transition state in this reaction conduce to the formation of anti- or syn-isomer. ${ }^{11,12}$ A plausible mechanism is shown in Scheme 3. If hydrogen bonding is formed between polyethyleneglycol, imine and enol form of cyclohexanone or methyl cyclohexanone, the aryl and phenyl group would be anti- to each other, so there is minimum steric repulsion between, methylene groups in cyclohexanones and aryl group, as well as polyethyleneglycol and $\mathrm{H}_{1}$. Therefore this transition state conduces to antiisomer. Because complete anti-selectivity is observed, we can conclude that powerful hydrogen bonding exists between polyethylene glycol, imine an enol form of cyclohexanones.

In summary, good stereoselectivity, high yields, facile operations, non-toxic solvent, easy workup, low catalyst loading and no-formation of by-products are merits of this procedure.
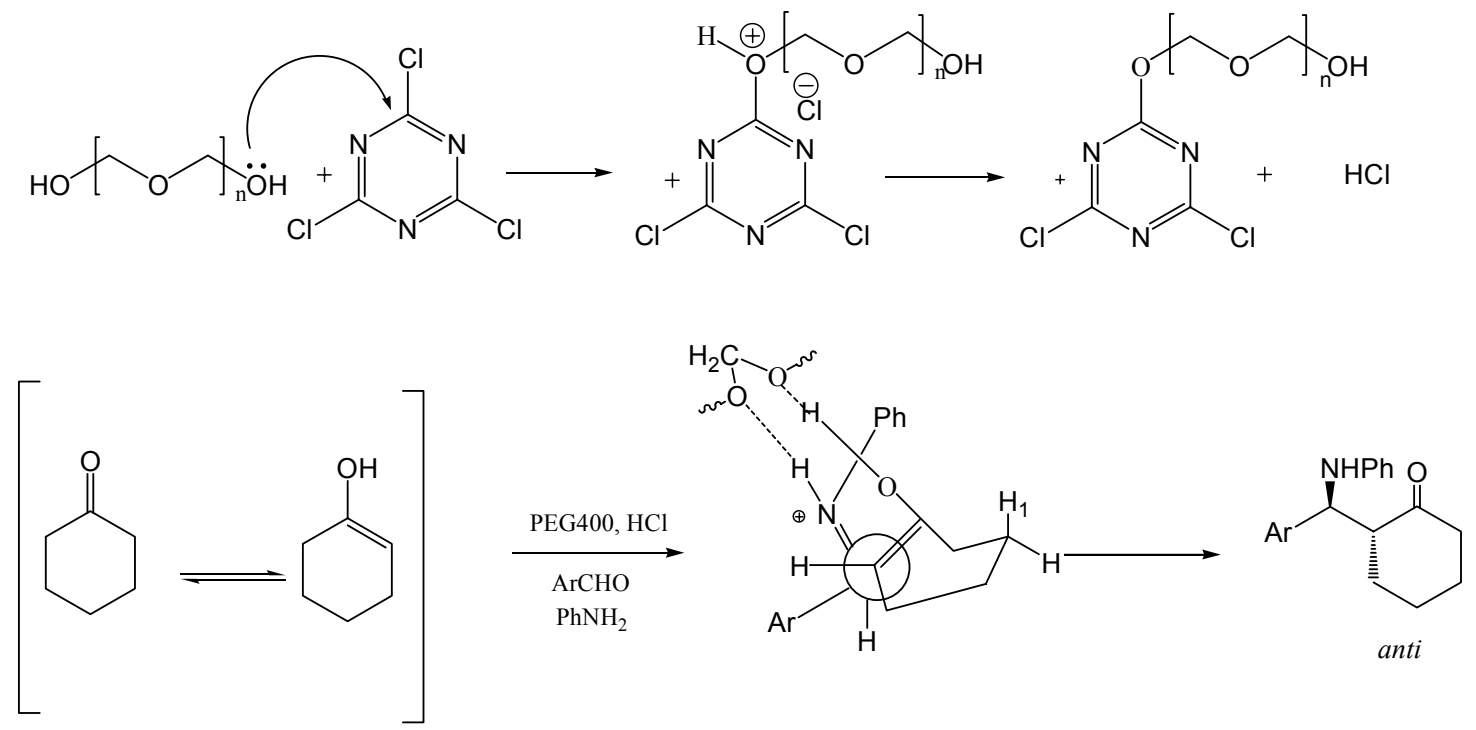

Scheme 3. A plausible mechanism.

\section{Experimental Section}

\section{General Procedures for the synthesis of $\beta$-carbonyl compounds}

A mixture of benzaldehyde $(2 \mathrm{mmol})$, aniline $(2 \mathrm{mmol})$, cyclohexanone $(2.2 \mathrm{mmol})$ and TCT $(0.027 \mathrm{mmol})$ was stirred in PEG $(3 \mathrm{ml})$ at room temperature for $1-2.5 \mathrm{~h}$. After completion of the reaction, water was added and mixture was filtered off. The filtrate was recrystallized from EtOH to give the pure $\beta$-amino ketone.

All the products were characterized by IR, ${ }^{1} \mathrm{H}$ NMR and ${ }^{13} \mathrm{C}$ NMR, and were identified by the comparison of the spectral data with those reported in literature. ${ }^{11-13}$

4-Methyl-2-((Phenyl(phenylamino)methyl)cyclohexanone (6). M.P. $=132-135^{\circ} \mathrm{C}$; IR $(\mathrm{KBr})$ : $v \max / \mathrm{cm}^{-1}: 3330,1702,1602,1497 ;{ }^{1} \mathrm{H}$ NMR $\left(300 \mathrm{MHz} ; \mathrm{CDCl}_{3} ; \mathrm{Me}_{4} \mathrm{Si}\right): \delta$ 7.41-7.38 (m, $\left.2 \mathrm{H}\right)$, 
7.32-7.2 (m, 2H), 7.12-7.07 (m, 1H), 6.68-6.63 (m, 3H), 6.59-6.55 (m, 2H), 5.08 (s, br, 1H), 4.58 (d, $J=4.45 \mathrm{~Hz}$, anti, $0.95 \mathrm{H}), 2.93-3.01(\mathrm{~m}, 1 \mathrm{H}), 2.29-2.43(\mathrm{~m}, 2 \mathrm{H}), 2.07-1.93(\mathrm{~m}, 3 \mathrm{H}), 1.61-1.46$ $(\mathrm{m}, 1 \mathrm{H}), 1.46-1.33(\mathrm{~m}, 1 \mathrm{H}), 0.98(\mathrm{~d}, J=6.13 \mathrm{~Hz}, 3 \mathrm{H}) .{ }^{13} \mathrm{C} \mathrm{NMR}\left(75 \mathrm{MHz}, \mathrm{CDCl}_{3}\right): \delta 212.6$, 147.6, 141.8, 129.11, 128.3, 127.3, 126.86, 117.5, 113.7, 58.42, 56.7, 42.4, 40.89, 35.92, 32.2, 27, 21.1. Anal. Calc. for $\mathrm{C}_{20} \mathrm{H}_{23} \mathrm{NO}$; C 81.91, H 7.84, N 4.77, Found: C 81.90, H 7.83, N 4.79.

4-Methyl-2-((1-naphthyl(phenylamino)methyl)cyclohexanone (7). M.P. $=169-172^{\circ} \mathrm{C}$; IR (KBr): $v \max / \mathrm{cm}^{-1}: 3379,2965,1703,1520 .{ }^{1} \mathrm{H} \mathrm{NMR}\left(300 \mathrm{MHz} ; \mathrm{CDCl}_{3} ; \mathrm{Me}_{4} \mathrm{Si}\right): \delta 8.16(\mathrm{~d}$, $J=8.4 \mathrm{~Hz}, 1 \mathrm{H}), 7.94(\mathrm{~d}, J=9 \mathrm{~Hz}, 1 \mathrm{H}), 7.8(\mathrm{~d}, J=7.1 \mathrm{~Hz}, 1 \mathrm{H}), 7.76(\mathrm{~d}, J=8.15 \mathrm{~Hz}, 1 \mathrm{H}), 7.68-7.56$ $(\mathrm{m}, 2 \mathrm{H}), 7.48-7.43(\mathrm{~m}, 1 \mathrm{H}), 7.09-7.04(\mathrm{~m}, 2 \mathrm{H}), 6.7-6.56(\mathrm{~m}, 3 \mathrm{H}), 5.35(\mathrm{~d}, J=4.8 \mathrm{~Hz}$, anti, 0.99H), 2.41-2.35 (m, 3H), 2.04-1.94(m, 3H), 1.57-1.42 (m, 2H), 0.95 (d, J=6 Hz, 3H). ${ }^{13} \mathrm{C}$ NMR $\left(75 \mathrm{MHz}, \mathrm{CDCl}_{3}\right): \delta$ 213.2, 147.46, 137.1, 133.9, 131.3, 129.54, 129.15, 127.6, 126.3, 125.8, $125.3,125.02,122.09,117.4,113.5,56.4,54.8,43.1,33.3,28.2,25.1$. Anal. Calc. for $\mathrm{C}_{24} \mathrm{H}_{25} \mathrm{NO}$ : C 83.96, H 7.28, N 4.08 Found: C 83.90, H 7.16, N 4.06.

4-Methyl-2-((2-naphthyl(phenylamino)methyl)cyclohexanone (8). M.P. $=174-178^{\circ} \mathrm{C} ; \quad I R$ $(\mathrm{KBr}): v \max / \mathrm{cm}^{-1}: 3400,2951,1699,1506,789 .{ }^{1} \mathrm{H} \mathrm{NMR}\left(300 \mathrm{MHz} ; \mathrm{CDCl}_{3} ; \mathrm{Me}_{4} \mathrm{Si}\right): \delta 7.84-$ $7.77(\mathrm{~m}, 4 \mathrm{H}), 7.55(\mathrm{~d}, \mathrm{~d}, J=1.7 \mathrm{~Hz}, J=8.5 \mathrm{~Hz}, 1 \mathrm{H}), 7.5-7.4(\mathrm{~m}, 2 \mathrm{H}), 7.11-7.04(\mathrm{~m}, 2 \mathrm{H}), 6.67-6.59$ $(\mathrm{m}, 3 \mathrm{H}), 5.27$ (s, br, 1H, ), 4.75 (d, $J=4.73$, anti, 0.99H), 3.07-3.03 (m, 1H), 2.41-2.35 (m, 2H), 2.04-1.94 (m, 3H), 1.57-1.42 (m, 2H), 0.96(d, $J=6.16 \mathrm{~Hz}, 3 \mathrm{H}) .{ }^{13} \mathrm{C}$ NMR $\left(75 \mathrm{MHz}, \mathrm{CDCl}_{3}\right): \delta$ 212.5, 147.4, 139.3, 133.28, 132.67, 129.1, 128.06, 127.9, 127.5, 126.3, 125.6, 125.57, 117.7, $114.2,113.9,113.5,70.5,58.7,56.1,42.4,40.8,35.9,32.2,21.1$. Anal. Calc. for $\mathrm{C}_{24} \mathrm{H}_{25} \mathrm{NO}: \mathrm{C}$ 83.96, H 7.28, N 4.08 Found: C 83.96, H 7.29, N 4.09.

4-Methyl-2-((2-chloro(phenylamino)methyl)cyclohexanone (9). M.P. $=146-147^{\circ} \mathrm{C}$; IR (KBr): $v \max / \mathrm{cm}^{-1}: 3390,1698,1600,1500,809 .{ }^{1} \mathrm{H}$ NMR $\left(300 \mathrm{MHz} ; \mathrm{CDCl}_{3} ; \mathrm{Me}_{4} \mathrm{Si}\right): \delta 7.58(\mathrm{~d}, \mathrm{~d}$, $J=1.9 \mathrm{~Hz}, J=7.5 \mathrm{~Hz}, 1 \mathrm{H}), 7.34$ (d,d, $J=\mathrm{Hz}, J=7.6 \mathrm{~Hz}, 1 \mathrm{H}), 7.26-7.07$ (m, 4H), 6.67-6.62 (m, 1H), 6.55-6.51 (m, 2H), $5.14(\mathrm{~d}, J=9 \mathrm{~Hz}$, anti, $0.99 \mathrm{H}, \mathrm{CH}), 4.72(\mathrm{~s}, \mathrm{br}, 1 \mathrm{H}), 3.6(\mathrm{~d}, J=2.34,1 \mathrm{H}), 2.43-$ 2.29 (m, 2H), 2.07-1.93 (m, 3H), 1.61-1.46 (m, 1H), 1.46-1.33 (m,1H), 1.04 (d, J=6.6Hz, 3H);

${ }^{13} \mathrm{C} \mathrm{NMR}\left(75 \mathrm{MHz}, \mathrm{CDCl}_{3}\right.$ ): $\delta$ 213.8, 146.6, 134, 129.3, 129.1, 128.6, 128.4, 127.6, 126.6, 117.9, 113.4, 55.3, 54.1, 39.08, 36.9, 34.7, 27.7, 20.2. Anal. Calc. for $\mathrm{C}_{20} \mathrm{H}_{22} \mathrm{NOCl}$ : C 73.28, $\mathrm{H} \mathrm{6.71,} \mathrm{N}$ 4.27 Found: C 73.29, H 6.72, N 4.25.

\section{References and Notes}

1. (a) Arend, M.; Westermnn, B. Angew. Chem., Int. Ed. 1998, 37, 1044. (b) Kobayashi, S.; Ishitani, H. Chem. Rev. 1999, 99, 1069. (c) Mannich, C.; Krosche, W. Arch. Pharm. 1912, $250,647$.

2. (a) Muller, R.; Goesmann, H.; Waldmann, H. Angew. Chem., Int. Ed. 1999, 38, 184. (b) Suginome, M.; Uehlin, L.; Murakami, M. J. Am. Chem. Soc. 2004, 126, 13196. (c) Notz, W.; Tanaka, F.; Watanabe, S.-I.; Chowdari, N. S.; Turner, J. M.; Thayumanavan, R.; Barbas, C. F. J. Org. Chem. 2003, 68, 9624 and references cited therein. 
3. (a) Josephsohn, N. S.; Snapper, M. L.; Hoveyda, A. H. J. Am. Chem. Soc. 2004, 126, 3734. (b) Lou, S.; Taoka, B. M.; Ting, A.; Schaus, S. E. J. Am. Chem. Soc. 2005, 127, 11256. (c) Ollevier, T.; Nadeau, E. J. Org. Chem. 2004, 69, 9292. (d) Joshi, N. S.; Whitaker, L. R.; Francis, M. B. J. Am. Chem. Soc. 2004, 126, 15942. (e) Xu, L.-W.; Xia, C. -G.; Li, L. J. Org. Chem. 2004, 69, 8482. (f) Notz, W.; Tananka, F.; Barbas, C. F. Acc. Chem. Res. 2004, 37, 580.(g) Hamada, T.; Manabe, K.; Kobayashi, S. J. Am. Chem. Soc. 2004,126, 7768. (h) Ibrahem, I.; Zou, W.; Engqvist, M.; Xu, Y.; Córdova, A. Chem. Eur. J. 2005, 11, 7024.(i) Córdova, A. Acc. Chem. Res. 2004, 37, 102. (j). Córdova, A. Chem. Eur. J. 2004, 10, 1987. (k) Chowdari, N. S.; Suri, J. T.; Barbas, C. F. Org. Lett. 2004, 6, 2507(1) Josephsohn, N. S.; Carswell, E. L.; Snapper, M. L.; Hoveyda, A. H. Org. Lett. 2005, 7, 2711.

4. (a) Kobayashi, S.; Matsubara, R.; Kitagawa, H. Org. Lett. 2002, 4, 143. (b) Ueno, M.; Ishitani, H.; Kobayashi, S. Org. Lett. 2002, 4, 3395. (c) Badorrey, R.; Cativiela, C.; Diaz-deVillegas, M. D.; Ga'lvez, J. A. Tetrahedron Lett. 2003, 44, 9189. (d) Periasamy, M.; Suresh, S.; Ganesan, S. S. Tetrahedron Lett. 2005, 46, 5521. (e) Jacobsen, M. F.; Ionita, L.; Skrydstrup, T. J. Org. Chem. 2004, 69, 680. (f) Chung, W. J.; Omote, M.; Welch, J. T. J. Org. Chem. 2005, 70, 7784. (g) Pandey, G.; Singh, R. P.; Garg, A.; Singh, V. K. Tetrahedron Lett. 2005, 46, 2137.

5. (a) Kobayashi, S.; Ishitani, H. J. Chem. Soc., Chem. Commun. 1995, 1379 (b) Wang, L.; Han, J.; Sheng, J.; Tian, H.; Fan, Z. Catal. Commun. 2005, 6, 201.

6. Ishitani, H.; Ueno, M.; Kobayashi, S. J. Am. Chem. Soc. 1997, 119, 7153.

7. (a) List, B. J. Am. Chem. Soc. 2000, 122, 9336. (b) List, B.; Pojariiev, P.; Biller, W. T.; Martin, H. J. J. Am. Chem. Soc.2002, 124, 12964. (c) Notz, W.; Sakthivel, K.; Bui, T.; Zhong, G.; Barbas, III, C. F. Tetrahedron Lett. 2001, 42, 199.

8. (a) Sharma, G. V. M.; Janardhan Reddy, J.; Sree Lakshmi, P.; Radhs Krishna, P. Tetrahedron Lett. 2004, 45, 7729. (b) De Luca, L.; Giacomelli, G.; Porcheddu, A. Org. Lett. 2002, 4, 553; and references cited therein.

9. (a) Loh, T. P.; Liung, S. B. K. W.; Tan, K. L.; Wei, L. L. Tetrahedron 2000, 56, 3227 and references cited therein. (b) Gennari, C.; Venturini, I.; Gislon, F.; Schimperma, G. Tetrahedron Lett. 1987, 28, 227. (c) Guanti, G.; Narisano, E.; Banfi, L. Tetrahedron Lett. 1987, 28, 4331.

10. Ranu, B. C.; Samanta, S.; Guchhait, S. K. Tetrahedron 2002, 58, 983.

11. Wu, H.; Shen, Y.; Fan, L.-Y.; Wan, Y.; Zhang, P.; Chen, C.-F.; Wang, W.-X. Tetrahedron, 2007, 63, 2404.

12. Bigdeli, M. A.; Nemati, F.; Mahdavinia, G. H; Tetrahedron Lett, 2007, 48, 6801.

13. (a) Yang, Y.-Y.; Shou, W.-G.; Wang, Y.-G. Tetrahedron, 2006, 10079. (b) Azizi, N.; Torkian, L.; Saidi, M. R. Org. Lett. 2006, 8, 2079. 